\title{
Key genes associated with pancreatic cancer and their association with outcomes: A bioinformatics analysis
}

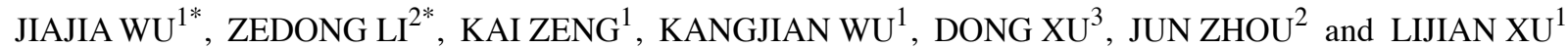 \\ ${ }^{1}$ Department of General Surgery, The Second Affiliated Hospital of Nanjing Medical University, Nanjing, Jiangsu 210000; \\ ${ }^{2}$ Department of Minimally Invasive Surgery, The Second Xiangya Hospital, Central South University, Changsha, \\ Hunan 410011; ${ }^{3}$ Department of General Surgery, Gaochun People's Hospital, Nanjing, Jiangsu 211300, P.R. China
}

Received October 4, 2018; Accepted April 9, 2019

DOI: $10.3892 / \mathrm{mmr} .2019 .10321$

\begin{abstract}
Pancreatic cancer is a highly malignant neoplastic disease of the digestive system. In the present study, the dataset GSE62165 was downloaded from the Gene Expression Omnibus (GEO) database. GSE62165 contained the data of 118 pancreatic ductal adenocarcinoma samples (38 early-stage tumors, 62 lymph node metastases and 18 advanced tumors) and 13 control samples. Differences in the expression levels of genes between normal tissues and early-stage tumors were investigated. A total of 240 differentially expressed genes (DEGs) were identified using R software 3.5 (137 upregulated genes and 103 downregulated genes). Then, the differentially expressed genes were subjected to Gene Ontology and Kyoto Encyclopedia of Genes and Genomes analysis. The following 18 core genes were identified using Cytoscape, based on the protein-interaction network of DEGs determined using the online tool STRING: EGF, ALB, COL17A1, FN1, TIMP1, PLAU, PLA2G1B, IGFBP3, PLAUR, VCAN, COL1A1, PNLIP, CTRL, PRSS3, COMP, CPB1, ITGA2 and CEL. The pathways of the core genes were primarily associated with pancreatic secretion, protein digestion and absorption, and focal adhesion. Finally, survival analyses of core genes in pancreatic cancer were conducted using the UALCAN online database. It was revealed that $P L A U$ and $C O L 17 A 1$ were significantly associated with poor prognosis $(\mathrm{P}<0.05)$. The expression levels of genes in primary pancreatic cancer tissues were then compared; only one gene, COL17A1, was identified to be significantly differentially expressed. Finally, another dataset from GEO, GSE28735, was analyzed to verify the upregulated expression of COL17A1. Taken together, the results of the present study have indicated
\end{abstract}

Correspondence to: Professor Lijian Xu, Department of General Surgery, The Second Affiliated Hospital of Nanjing Medical University, 121 Jiangjiayuan Road, Gulou, Nanjing, Jiangsu 210000, P.R. China

E-mail: xulijian134@126.com

*Contributed equally

Key words: pancreatic cancer, bioinformatics analysis, prognosis that the expression of $C O L 17 A 1$ gene may be associated with the occurrence and development of pancreatic cancer.

\section{Introduction}

Pancreatic cancer is a highly malignant neoplasm of the digestive system that accounts for $>200,000$ deaths/year globally (1). The incidence of pancreatic cancer is low compared with that of lung, breast, colorectal and gastric cancers; however, it is associated with a very high mortality rate. It has been reported that the incidence of pancreatic cancer is very similar to the associated mortality rate; the reported 5-year survival rate of patients with pancreatic cancer is $<6 \%$ (2). The mortality rate of patients with pancreatic cancer ranks fourth among common cancers, and is predicted to rise to second within a decade (3). A number of factors have been identified as contributing to the etiopathogenesis of pancreatic cancer, including heredity, smoking, high-fat diet, chronic pancreatitis and consumption of nitrous acid compounds (4). Due to the latency of pancreatic cancer, the majority of patients are diagnosed at an advanced stage, when tumor tissue has already infiltrated the surrounding tissues and has formed distant metastases, decreasing the usefulness of surgical interventions (5). As a result of drug resistance, the efficacy of postoperative adjuvant therapy has also been very unsatisfactory (6). Carbohydrate antigen 19-9 (CA19-9) is the most frequently used marker for the clinical diagnosis of cancer; the reported sensitivity and specificity of CA19-9 for the diagnosis of pancreatic cancer is 69-93 and 46-98\%, respectively (7). Therefore, early diagnosis and treatment are important to improve the prognosis and survival of patients with pancreatic cancer.

At present, high-throughput sequencing is employed in a variety of contexts, such as the discovery of gene mutations and chromosomal translocations that are closely associated with the occurrence and development of tumors (8-10). High-throughput sequencing may be useful for the diagnosis of cancer and development of targeted therapies. These analyses may provide novel insights to guide subsequent research.

\section{Materials and methods}

Microarray data. The gene expression profile of GSE62165 (11) was downloaded from the GEO database (12). The data were 
created using the GPL13667 Affymetrix ${ }^{\circledR}$ Human Genome U219 array (Affymetrix; Thermo Fisher Scientific, Inc.). GSE62165 contained data on 118 pancreatic ductal adenocarcinoma (PDAC) samples and 13 control samples. Data were standardized using the robust multi-array average (RMA) algorithm using limma package (version 3.38.3) (13). In addition, a separate dataset, GSE28735 $(14,15)$, was used to verify the results. The expression profiles included 45 matched pairs of pancreatic tumor and adjacent non-tumor tissues from 45 patients with PDAC. The Cancer Genome Atlas (TCGA; http://cancergenome.nih.gov/) contains genomic sequencing data involving 33 species of cancer.

Identification of differentially expressed genes (DEGs). The limma package (version 3.38.3) (13) was used to identify DEGs between pancreatic cancer tissue and normal pancreatic tissue samples in R software (version 3.5; https://www.R-project.org). llog2 Fold Change (FC) $>3.0$ and adjusted P-value $<0.05$ were considered to be the threshold for differential gene identification.

Gene Ontology (GO) and Kyoto Encyclopedia of genes and genomes (KEGG) pathway analysis of DEGs. GO (http://www.geneontology.org/) and the KEGG (https://www. kegg.jp/) (16-19) were used to analyze the function of DEGs using the cluster Profiler R package (20). $\mathrm{P}<0.05$ was considered to indicate a statistically significant difference in functional enrichment analysis.

Core genes screening from the protein-protein interaction (PPI) network. A PPI network for the DEGs was generated using the STRING database (https://string-db.org/). Then, Cytoscape (version 3.6.1) (21) was employed, and a plug-in termed cytohubba (22) was integrated into the software. The plug-in provides 12 types of topological analysis methods [Maximal Clique Centrality, Maximum Neighborhood Component (MNC), Density of MNC, Degree, Edge Percolated Component, Bottleneck, EcCentricity, Closeness, Radiality, Betweenness, Stress and Clustering Coefficient). Using 12 analysis methods, we identified the top 18 genes as core genes.

Expression levels and survival analysis of core genes in pancreatic cancer. UALCAN (http://ualcan.path.uab. edu/index.html) (23) was employed to perform survival analysis based on the information of TCGA database. Survival analysis was performed via the Kaplan-Meier method using 18 identified core genes, based on their core gene expression levels in pancreatic adenocarcinoma (PAAD). $\mathrm{P}<0.05$ was considered to be statistically significant. The P-value was calculated using log-rank test. The 'scaled_estimate' column provided the potential transcripts produced by each gene. The 'scaled_estimate' was multiplied by $10^{6}$ to obtain a transcripts per million (TPM) expression value (24). Gene expression levels in tumor tissues exhibited notable inter-individual variability. High expression indicated that the TPM value was above the upper quartile value. Low expression indicated that the TPM value was equal or below the upper quartile value.

Verification of results. The findings from the bioinformatics analyses were validated using the dataset GSE28735 from the GEO database. The expression profiles included 45
Table I. Top 20 differentially expressed genes in early-stage pancreatic cancer tissues based on $\log 2 \mathrm{FC}$.

A, Upregulated genes

\begin{tabular}{llc}
\hline Gene symbol & Log2FC & Adjusted P-value \\
\hline COL1A1 & 5.9240335 & $3.17 \times 10^{-16}$ \\
KRT17 & 5.5334643 & $4.10 \times 10^{-12}$ \\
CEACAM5 & 5.3990511 & $1.66 \times 10^{-09}$ \\
S100P & 5.2665291 & $1.66 \times 10^{-13}$ \\
COL10A1 & 5.2258147 & $1.17 \times 10^{-17}$ \\
SERPINB5 & 5.1642588 & $5.50 \times 10^{-15}$ \\
GJB2 & 5.0747799 & $7.98 \times 10^{-18}$ \\
COL17A1 & 5.0501325 & $1.80 \times 10^{-11}$ \\
CXCL5 & 5.0384043 & $1.28 \times 10^{-11}$ \\
TMPRSS4 & 5.0203823 & $2.37 \times 10^{-16}$ \\
SDR16C5 & 4.9961337 & $3.16 \times 10^{-16}$ \\
CTHRC1 & 4.9626426 & $7.77 \times 10^{-20}$ \\
COL11A1 & 4.9350078 & $6.41 \times 10^{-17}$ \\
SLC6A14 & 4.8841916 & $2.47 \times 10^{-15}$ \\
MMP11 & 4.8824426 & $3.14 \times 10^{-16}$ \\
SULF1 & 4.721966 & $2.96 \times 10^{-17}$ \\
FN1 & 4.6424864 & $2.99 \times 10^{-16}$ \\
POSTN & 4.6415794 & $1.33 \times 10^{-16}$ \\
CCL18 & 4.5489901 & $1.41 \times 10^{-11}$ \\
MUC4 & 4.5022059 & $1.25 \times 10^{-09}$ \\
\hline
\end{tabular}

B, Downregulated genes

\begin{tabular}{llc}
\hline Gene symbol & Log2FC & Adjusted P-value \\
\hline SYCN & -6.6535946 & $2.74 \times 10^{-07}$ \\
SERPINI2 & -6.2894352 & $8.73 \times 10^{-09}$ \\
AQP8 & -6.2356139 & $2.11 \times 10^{-10}$ \\
AMY1A & -6.1790263 & $4.38 \times 10^{-10}$ \\
ALB & -6.1165814 & $2.16 \times 10^{-08}$ \\
CELA2A & -6.0845313 & $2.83 \times 10^{-06}$ \\
PNLIPRP1 & -6.0676353 & $6.52 \times 10^{-10}$ \\
CTRL & -5.9224624 & $1.73 \times 10^{-06}$ \\
PDIA2 & -5.9185276 & $4.65 \times 10^{-09}$ \\
CPA1 & -5.804844 & $5.21 \times 10^{-06}$ \\
TMED6 & -5.7967792 & $2.37 \times 10^{-10}$ \\
CELP & -5.7183603 & $1.15 \times 10^{-10}$ \\
AQP12A & -5.6598636 & $3.88 \times 10^{-14}$ \\
CUZD1 & -5.5766969 & $1.68 \times 10^{-06}$ \\
CELA2B & -5.5649112 & $2.23 \times 10^{-05}$ \\
CPA2 & -5.55513 & $3.43 \times 10^{-06}$ \\
CELA3A & -5.5508171 & $1.84 \times 10^{-05}$ \\
GP2 & -5.5087922 & $1.20 \times 10^{-06}$ \\
ERP27 & -5.4765153 & $7.39 \times 10^{-09}$ \\
CPA1 & -5.4417426 & $1.81 \times 10^{-05}$ \\
\hline
\end{tabular}

$\log 2 \mathrm{FC}, \log 2$ fold change.

matched pairs of pancreatic tumor and adjacent non-tumor tissues from 45 patients with PDAC. The online analysis tool 


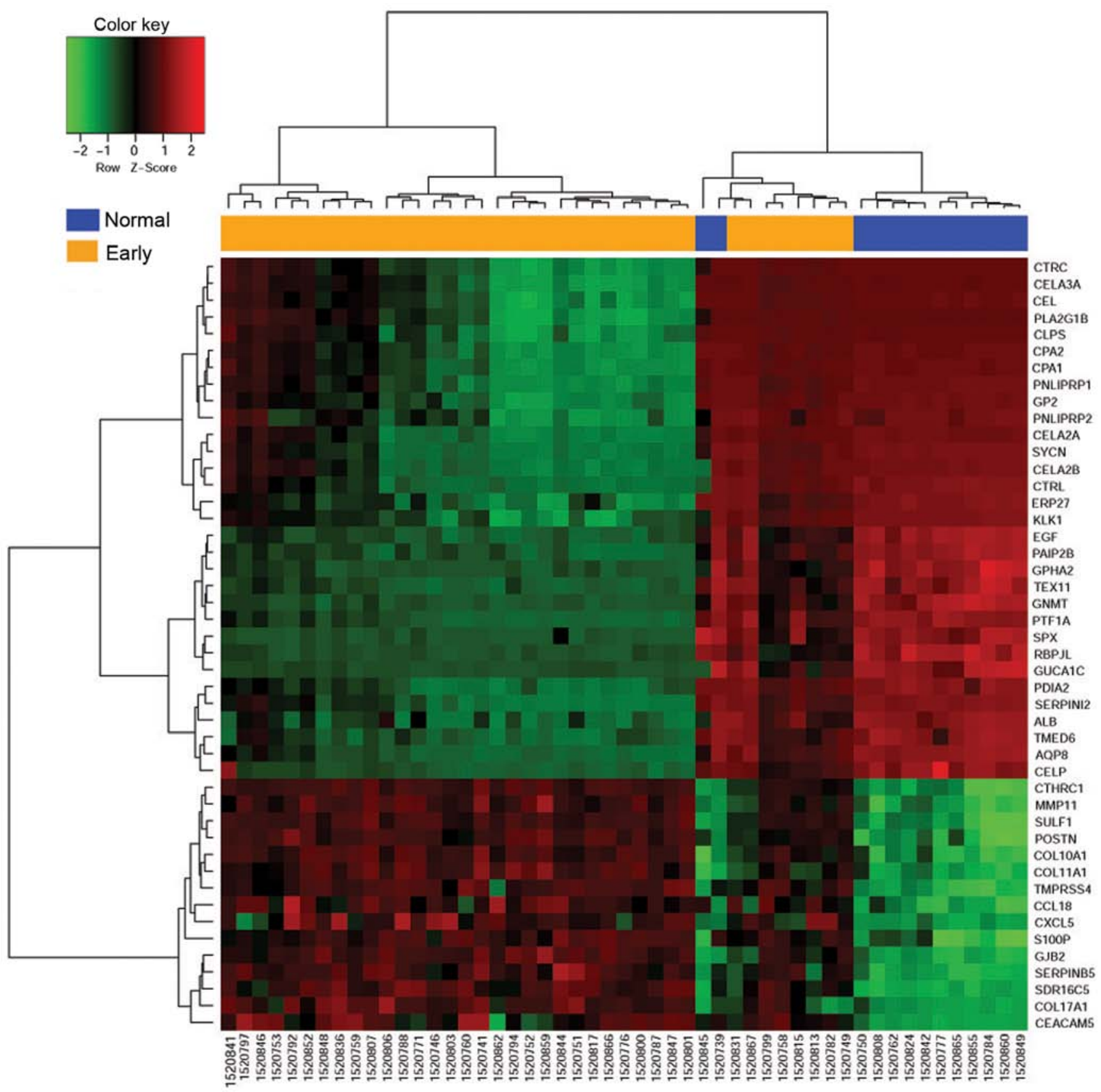

Figure 1. Heat map of gene expression in pancreatic ductal adenocarcinoma tissues and healthy controls. The expression levels of various genes in 51 samples (38 early-stage tumor samples and 13 controls) are presented. Green indicates downregulated expression; red indicates upregulated expression; black indicates no significant difference in expression.

GEO2R (https://www.ncbi.nlm.nih.gov/geo/geo2r/) was used to determine the expression of DEGs. We further verified the expression of COL17A1.

\section{Results}

Analysis of DEGs. The selected chipset GSE62165 included 118 PDAC samples and 13 control samples. Differences in gene expression profiles were analyzed using 38 early-stage tumors and 13 normal tissues. A total of 240 DEGs (adjusted P-value $<0.05 ; \mid \log _{2} \mathrm{FCl} \geq 3.0$ ) were identified using $R$ version 3.5 software, including 137 upregulated genes and 103 downregulated genes (Table I). The heat map of genes with upregulated expression is presented in Fig. 1. A volcanic map of all genes is presented in Fig. 2.

Enrichment analysis of DEGs. To investigated the distribution of DEGs, GO and KEGG analysis of upregulated and downregulated genes was conducted. GO analysis revealed that the 'biological processes' (BPs) of upregulated genes mainly included extracellular matrix organization, extracellular structure organization and collagen catabolic process. 'Molecular functions' (MFs) of upregulated DEGs primarily included extracellular matrix structural constituents, glycosaminoglycan binding and cytokine activity. For the "cell components' (CCs) identified by GO analysis, proteinaceous 


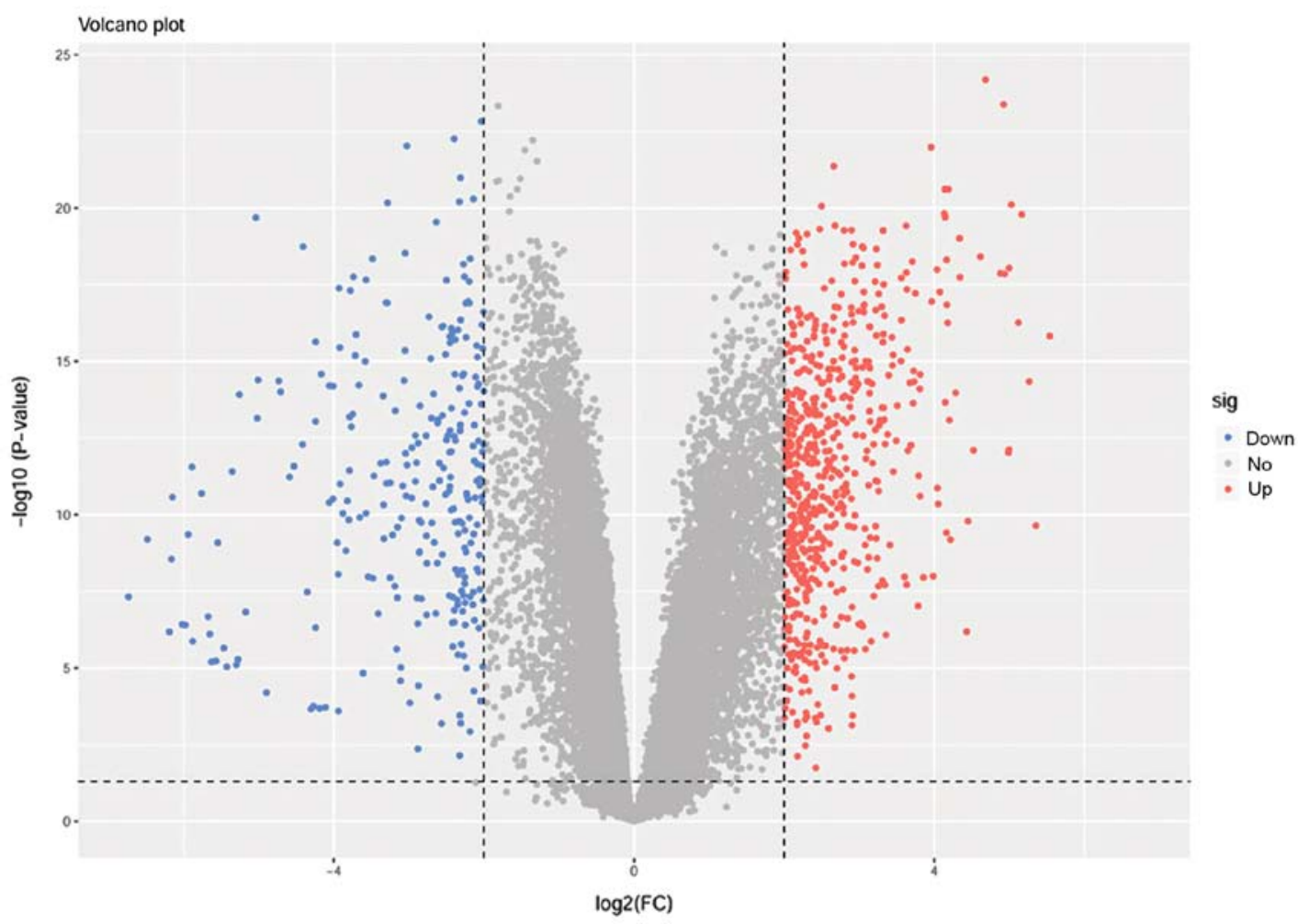

Figure 2. Volcano plot of the expression of genes in patients with early-stage pancreatic ductal adenocarcinoma. The expression of all identified genes in tumor tissues compared with in healthy control samples is presented. Blue indicates downregulated genes; red indicates upregulated genes; grey indicates genes that were not significantly differentially expressed. FC, fold change.

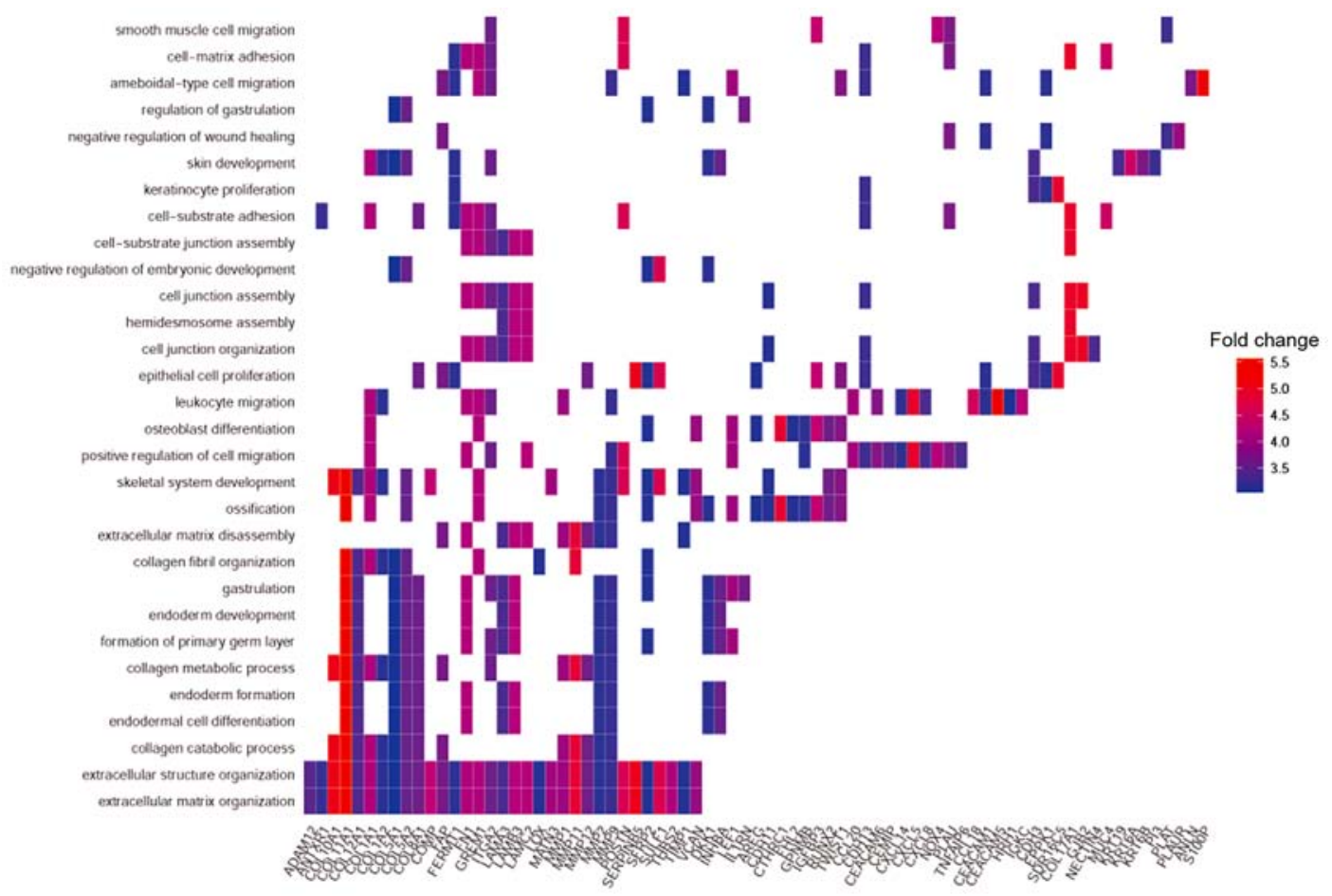

Figure 3. Functions of genes upregulated in pancreatic cancer tissues. Heat plot of the cell components, molecular functions and biological processes of upregulated genes in pancreatic cancer tissues, as identified by Gene Ontology analysis.

extracellular matrix, extracellular matrix component and endoplasmic reticulum lumen were the most prominent (Table II). For downregulated DEGs, the main enriched BPs were digestion, lipid digestion and sulfur amino acid metabolic process, whereas the primary MFs were exopeptidase activity, serine-type endopeptidase activity and serine-type 
Table II. GO analysis of differentially expressed genes in pancreatic cancer.

A, Upregulated genes

\begin{tabular}{|c|c|c|c|c|}
\hline Category & ID & Description & Count & P-value \\
\hline GOBP & GO:0030198 & Extracellular matrix organization & 22 & $9.83 \times 10^{-21}$ \\
\hline GOBP & GO:0043062 & Extracellular structure organization & 22 & $1.05 \times 10^{-20}$ \\
\hline GOBP & GO:0030574 & Collagen catabolic process & 10 & $2.16 \times 10^{-13}$ \\
\hline GOBP & GO:0044243 & Multicellular organismal catabolic process & 10 & $6.89 \times 10^{-13}$ \\
\hline GOBP & GO:0032963 & Collagen metabolic process & 11 & $4.16 \times 10^{-12}$ \\
\hline GOMF & GO:0005201 & Extracellular matrix structural constituent & 8 & $1.09 \times 10^{-09}$ \\
\hline GOMF & GO:0005539 & Glycosaminoglycan binding & 7 & $2.85 \times 10^{-05}$ \\
\hline GOMF & GO:0005125 & Cytokine activity & 7 & $3.50 \times 10^{-05}$ \\
\hline GOMF & GO:0008009 & Chemokine activity & 4 & $4.62 \times 10^{-05}$ \\
\hline GOMF & GO:1901681 & Sulfur compound binding & 7 & $4.91 \times 10^{-05}$ \\
\hline GOCC & GO:0005578 & Proteinaceous extracellular matrix & 20 & $1.05 \times 10^{-17}$ \\
\hline GOCC & GO:0044420 & Extracellular matrix component & 10 & $4.54 \times 10^{-11}$ \\
\hline GOCC & GO:0005788 & Endoplasmic reticulum lumen & 13 & $1.70 \times 10^{-10}$ \\
\hline GOCC & GO:0005581 & Collagen trimer & 8 & $2.20 \times 10^{-09}$ \\
\hline GOCC & GO:0098644 & Complex of collagen trimers & 5 & $1.32 \times 10^{-08}$ \\
\hline
\end{tabular}

B, Downregulated genes

\begin{tabular}{|c|c|c|c|c|}
\hline Category & ID & Description & Count & P-value \\
\hline GOBP & GO:0007586 & Digestion & 10 & $7.20 \times 10^{-10}$ \\
\hline GOBP & GO:0044241 & Lipid digestion & 5 & $1.00 \times 10^{-08}$ \\
\hline GOBP & GO:0000096 & Sulfur amino acid metabolic process & 4 & $1.27 \times 10^{-05}$ \\
\hline GOBP & GO:0009235 & Cobalamin metabolic process & 3 & $6.62 \times 10^{-05}$ \\
\hline GOBP & GO: 1901605 & $\alpha$-Amino acid metabolic process & 6 & $1.76 \times 10^{-4}$ \\
\hline GOMF & GO:0008238 & Exopeptidase activity & 8 & $7.37 \times 10^{-09}$ \\
\hline GOMF & GO:0004252 & Serine-type endopeptidase activity & 10 & $2.66 \times 10^{-08}$ \\
\hline GOMF & GO:0008236 & Serine-type peptidase activity & 10 & $7.39 \times 10^{-08}$ \\
\hline GOMF & GO:0008235 & Metalloexopeptidase activity & 6 & $8.31 \times 10^{-08}$ \\
\hline GOMF & GO:0017171 & Serine hydrolase activity & 10 & $8.76 \times 10^{-08}$ \\
\hline
\end{tabular}

GO, Gene Ontology; MF, molecular function; CC, cell component; BP, biological process.

peptidase activity (Table II). Figs. 3 and 4 present the associations between genes and enrichment results, indicating the genes that were highly changed between the two conditions.

Table III presents KEGG pathway analysis of the DEGs, revealing that the upregulated genes were mainly located in extracellular matrix (ECM)-receptor interaction, protein digestion and absorption, and focal adhesion pathways. Conversely, downregulated genes were primarily located in pancreatic secretion, protein digestion and absorption, and fat digestion and absorption pathways. Figs. 5 and 6 present the distribution of the major KEGG pathways generated using clusterProfiler. It was observed that ECM-receptor interactions (Fig. 5) and pancreatic secretion (Fig. 6) were the pathways most enriched with up- and downregulated DEGs, respectively.

Screening of core genes in the PPI. Based on the information in the STRING database and using 12 types of calculation methods in Cytoscape, the following 18 core genes were identified: EGF, ALB, COL17A1, FN1, TIMP1, PLAU, PLA2G1B, IGFBP3, PLAUR, VCAN, COLIA1, PNLIP, CTRL, PRSS3, $C O M P, C P B 1, I T G A 2$ and $C E L$. These core genes were associated with each other and may exhibit synergistic effects in the development of pancreatic cancer (Fig. 7). According to the previous enrichment analysis, the core genes, were mainly located in pancreatic secretion, protein digestion and absorption, and focal adhesion pathways.

Gene expression level and survival analysis. Notably, COL17A1 and PLAU genes were the only genes associated with survival. Following the identification of core genes, survival analysis for PAAD was performed using UALCAN. $P L A U$ [which encodes the serine protease urokinase-type plasminogen activator (uPA); Fig. 8] and COL17A1 [which encodes collagen type XVII $\alpha 1$ chain (COL17A1); Fig. 9] 
Table III. KEGG pathway analysis of differentially expressed genes in pancreatic cancer.

A, Upregulated genes

\begin{tabular}{llll}
\hline ID & \multicolumn{1}{c}{ Description } & Count & P-value \\
\hline hsa04512 & Extracellular matrix-receptor interaction & 7 & $2.23 \times 10^{-07}$ \\
hsa04974 & Protein digestion and absorption & 7 & $4.25 \times 10^{-07}$ \\
hsa04510 & Focal adhesion & 7 & $8.15 \times 10^{-05}$ \\
hsa04657 & Interleukin-17 signaling pathway & 5 & $1.32 \times 10^{-4}$ \\
hsa05146 & Amebiasis & 5 & $1.53 \times 10^{-4}$ \\
\hline
\end{tabular}

B, Downregulated genes

\begin{tabular}{llrr}
\hline ID & \multicolumn{1}{c}{ Description } & Count & P-value \\
\hline hsa04972 & Pancreatic secretion & 13 & $3.63 \times 10^{-16}$ \\
hsa04974 & Protein digestion and absorption & 8 & $1.03 \times 10^{-08}$ \\
hsa04975 & Fat digestion and absorption & 6 & $4.10 \times 10^{-08}$ \\
hsa00561 & Glycerolipid metabolism & 4 & $2.27 \times 10^{-4}$ \\
hsa00260 & Glycine, serine and threonine metabolism & 3 & $1.01 \times 10^{-3}$ \\
\hline
\end{tabular}

KEGG, Kyoto Encyclopedia of Genes and Genomes.

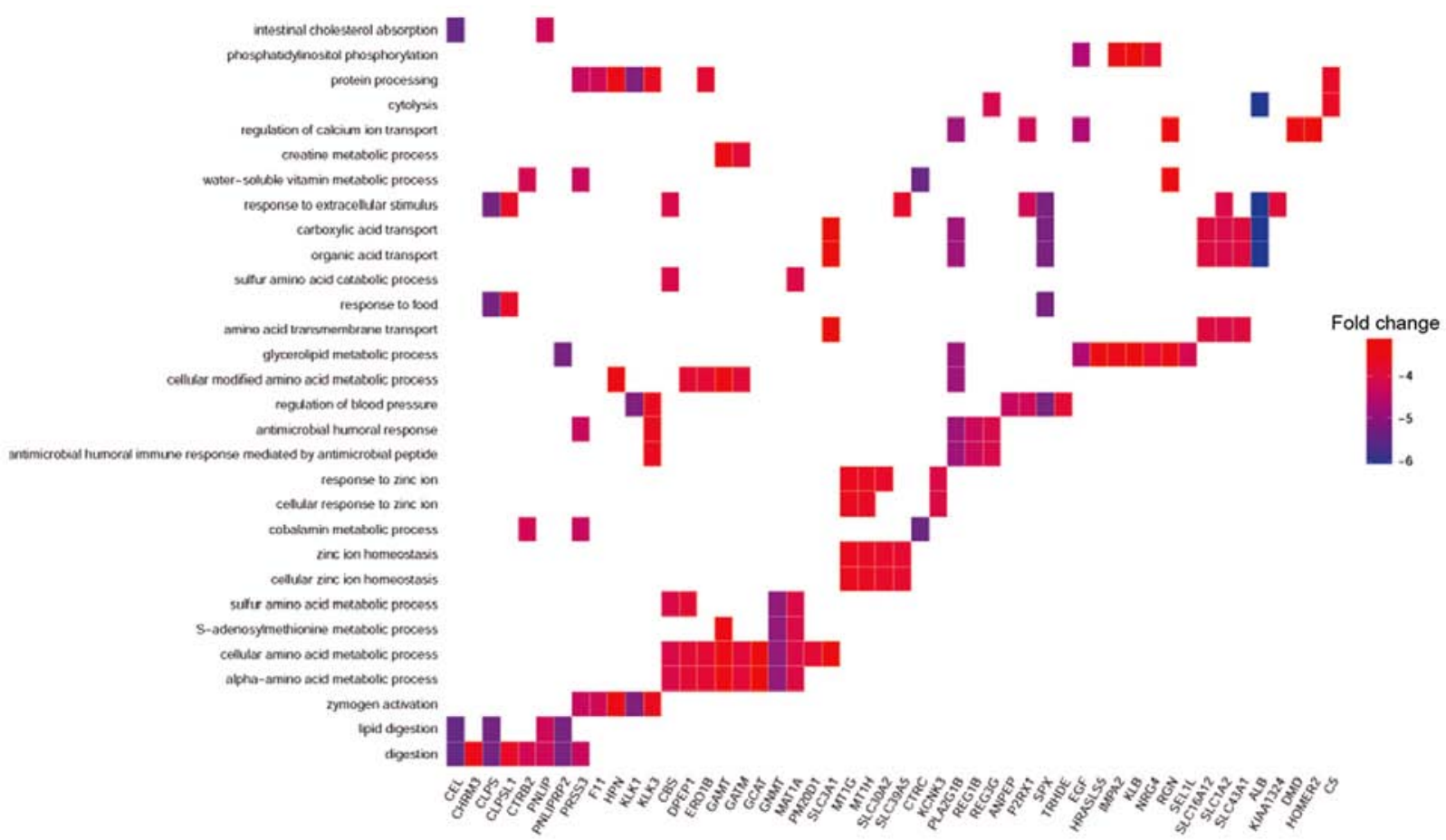

Figure 4. Functions of genes downregulated in pancreatic cancer tissues. Heat plot of the molecular functions and biological processes of downregulated genes in pancreatic cancer tissues, as identified by Gene Ontology analysis.

were demonstrated to be significantly associated with survival $(\mathrm{P}<0.05)$. Subsequently, the expression levels of genes in primary pancreatic cancer were compared; only one gene was identified to be significantly differentially expressed, COL17A1, whereas PLAU was not significantly differentially expressed. The expression levels of COL17Al were analyzed in TCGA database, and the results were consistent with those of the aforementioned differential gene analysis; $C O L 17 A 1$ was significantly upregulated in PAAD tumor tissues compared with normal tissues $\left(\mathrm{P}=1.62 \times 10^{-12}\right.$; Fig. 10).

Verification of COL17A1. Differences in gene expression between 45 pancreatic cancer patients and 45 normal pancreatic tissues were analyzed. In particular, the expression level 


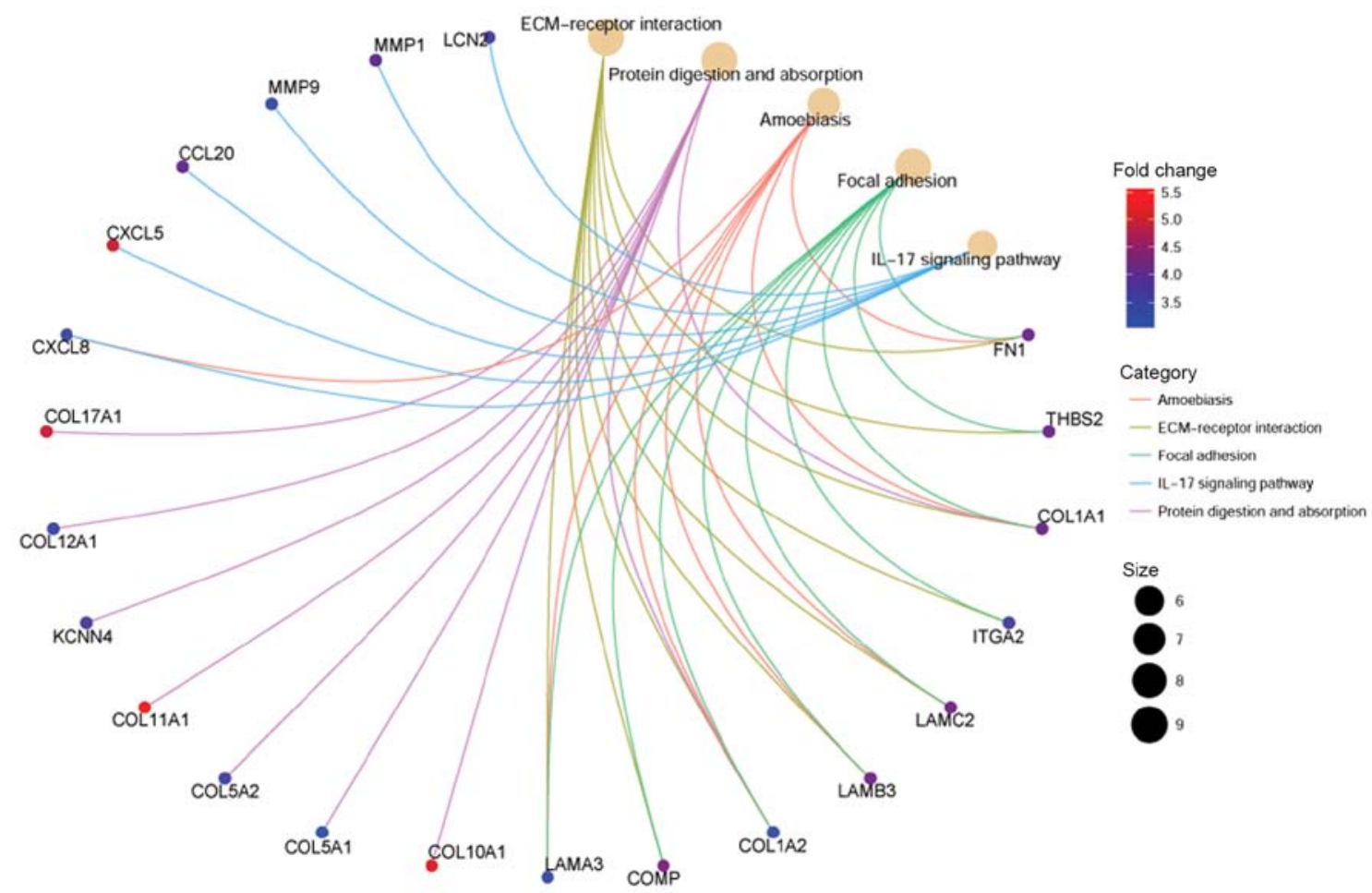

Figure 5. Pathways enriched with genes upregulated in pancreatic cancer. Net plot of the pathways enriched with genes identified as upregulated in pancreatic cancer tissues, as identified by Kyoto Encyclopedia of Genes and Genomes pathway analysis. ECM, extracellular matrix; IL-17, interleukin-17.

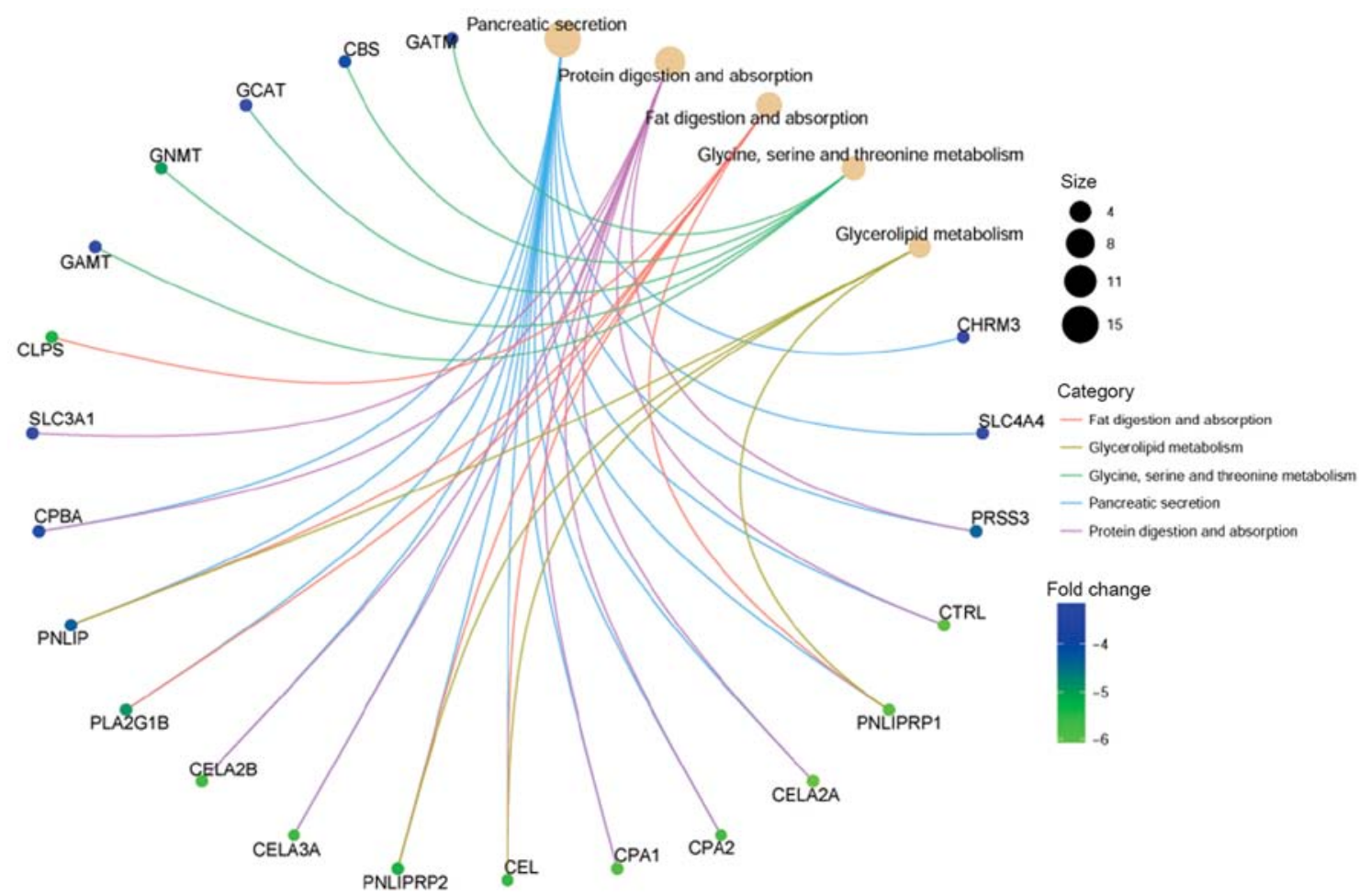

Figure 6. Pathways enriched with genes downregulated in pancreatic cancer. Net plot of the pathways enriched with genes identified as downregulated in pancreatic cancer tissues, as identified by Kyoto Encyclopedia of Genes and Genomes pathway analysis.

of COL17A1 was investigated. The results of the analysis to verify the importance of COL17Al are presented in Table IV; it was observed that COL17Al was significantly upregulated in pancreatic tumor tissue in the two GEO databases.

\section{Discussion}

The incidence of pancreatic cancer and the associated mortality rates have exhibited an increasing trend in previous years (3). 


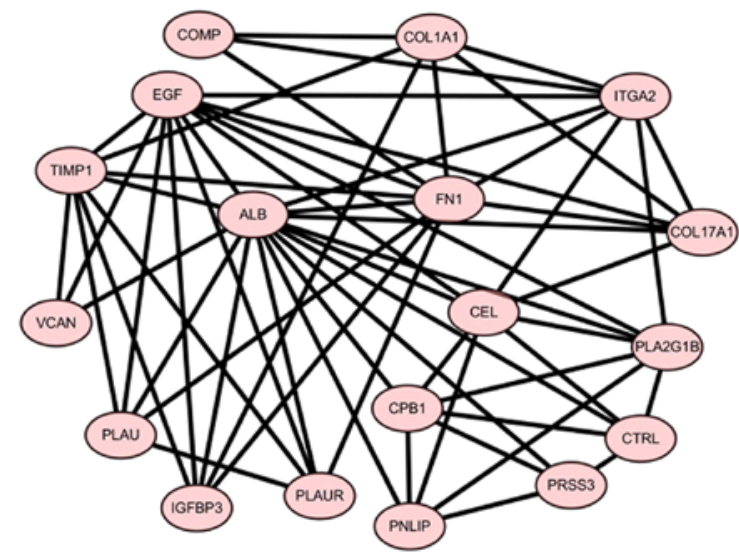

Figure 7. Protein-protein interaction network of the 18 identified core genes.

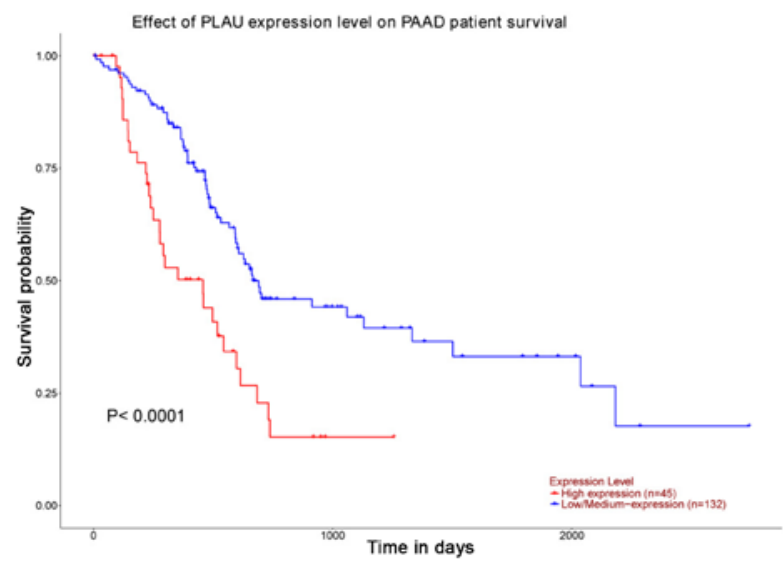

Figure 8. Survival analysis of $P L A U$ in PAAD. Kaplan-Meier analysis of the association between the expression of $P L A U$ and the overall survival of patients with PAAD. PAAD, pancreatic adenocarcinoma; PLAU, gene encoding urokinase-type plasminogen activator.

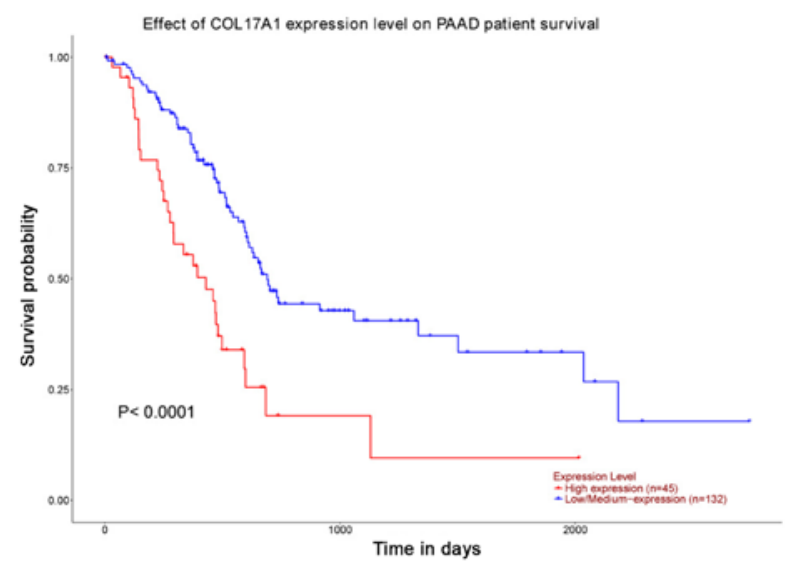

Figure 9. Survival analysis of COL17A1 in PAAD. Kaplan-Meier analysis of the association between the expression of COL17A1 and the overall survival of patients with PAAD. PAAD, pancreatic adenocarcinoma; COL17Al, gene encoding collagen type XVII $\alpha 1$ chain.

Studies have reported that patients with pancreatic cancer survive for only 4 months on average without treatment; even in patients who undergo treatment, the survival is not significantly extended (25). Therefore, accurate early diagnosis of
Table IV. Differential expression of COL17A1 in pancreatic cancer tissues in two databases.

\begin{tabular}{lllc}
\hline Database & Gene & Log2FC & Adjusted P-value \\
\hline GSE62165 & COL17A1 & 5.0501325 & $1.8 \times 10^{-11}$ \\
GSE28735 & COL17A1 & 1.893626 & $6.56 \times 10^{-13}$ \\
\hline
\end{tabular}

Log2FC, Log2 fold change; COL17A1, collagen type XVII $\alpha 1$ chain.

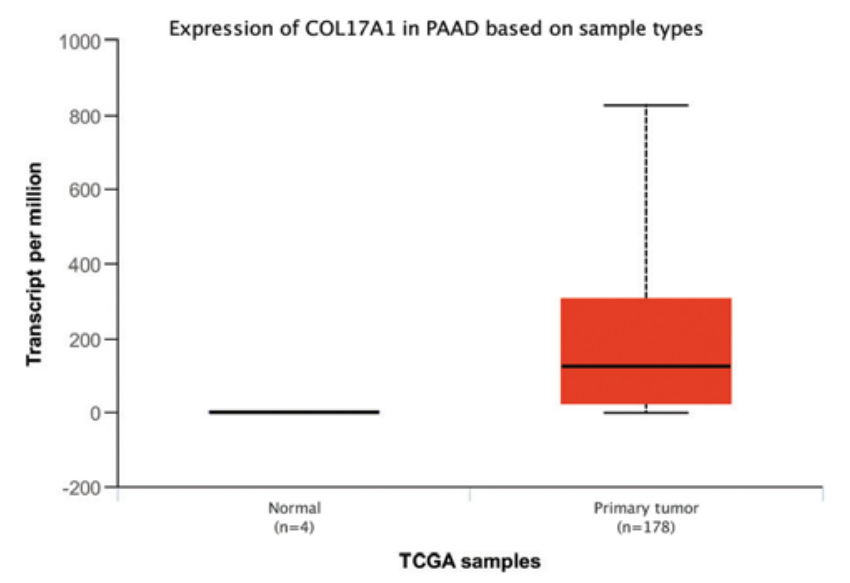

Figure 10. Expression levels of COL17A1 in PAAD and normal tissues. The expression of COL17A1 was compared between PAAD primary tumor and normal control tissues, based on data from TCGA. PAAD, pancreatic adenocarcinoma; COL17Al, gene encoding collagen type XVII $\alpha 1$ chain; TCGA, The Cancer Genome Atlas.

pancreatic cancer and the development of effective targeted therapy is of major importance.

A previous study identified core genes in pancreatic cancer that were reported to be of diagnostic relevance (26). In the present study, the chipset GSE62165 from the GEO was analyzed, containing data of 118 PDAC and 13 normal pancreatic tissues (11). Differences in gene expression levels were only compared between normal tissues and early-stage tumor tissue. A total of 240 DEGs (137 upregulated and 103 downregulated) were identified using R, and GO (27) and KEGG pathway analyses of DEGs revealed the locations and functions of DEGs. Upregulated genes were mainly located in the ECM and collagen trimers, and were involved in ECM organization and ECM-receptor interactions, focal adhesion, and protein digestion and absorption. Conversely, downregulated genes were mainly enriched in digestion and exopeptidase activity pathways. A PPI network was built, and 18 core genes were identified; the prognostic value of these genes for patients with pancreatic cancer was analyzed using UACLAN. PLAU and COL17A1 were significantly associated with poorer survival; it was then revealed using data from TCGA that COL17Al was significantly upregulated in pancreatic cancer tissues compared with control tissues, consistent with the results of the differential gene analysis. It was predicted that these two genes may be associated with the proliferation, invasion and metastasis of pancreatic cancer.

PLAU encodes a serine protease, uPA (28). Following GO and KEGG analyses, the functional enrichment of PLAU was 
investigated. PLAU is mainly involved in the regulation of cell motility, cellular component movement and locomotion (29). It is primarily expressed in the endoplasmic reticulum lumen and invadopodium (30). PLAU plays a key role in regulating cell migration and adhesion during tissue regeneration and intracellular signaling (31). Increased expression of COL17A1 leads to tumor cell invasion and metastasis of tumor cells to surrounding tissues (32). PLAU is involved in predicting the survival rate of patients with gastric cancer (33). It may serve an important role in the invasion and metastasis of pancreatic cancer cells (34); however, the specific pathways involved are yet to be determined. It is hypothesized that PLAU may serve an important role in the diagnosis and treatment of pancreatic cancer in the future.

COL17A1 is mainly located in the extracellular matrix and collagen trimmers (35). Extracellular matrix molecules, including proteoglycan and fibrin, have been reported to affect the growth, migration and differentiation of cells (36). A study showed that COL17A1 can inhibit the migration and invasion of breast cancercells, acting as a 53 transcriptional target gene (37). A previous study has reported that the extracellular matrix is closely associated with the metastasis of breast cancer (38). High levels of collagen in breast and colorectal cancers have been associated with tumor invasion $(39,40)$. A previous study that employed the minimum-redundancy-maximum-relevance method also identified COL17A1 as a core gene of pancreatic cancer (26); however, in the present study, the upregulated expression of COL17A1 in pancreatic cancer was verified in multiple datasets, and its effects on patient survival were determined. Survival analysis using UACLAN based on data from TCGA revealed that the expression levels of $C L O 17 A 1$ were closely associated with the survival of patients with pancreatic cancer, and that CLO17Al was highly expressed in primary pancreatic tumor tissues. The present findings suggested that the expression of COL17A1 is associated with the occurrence and development of pancreatic cancer. Therefore, this bioinformatics analysis may provide novel insight for future studies investigating the pathogenesis of pancreatic cancer.

However, the present study presented certain limitations. In examining the expression level of COL17A1, only four normal samples were investigated, and further studies examining a high number of control samples are required to confirm the present results.

\section{Acknowledgements}

Not applicable.

\section{Funding}

The present work was supported by The 'Six Talents Summit' Project in Jiangsu Province, miR-203 targets Survivin to upregulate the expression of Caspase-3 and promote the apoptosis of pancreatic cancer cells (grant no. WAW-008).

\section{Availability of data and materials}

The datasets used and/or analyzed in the present study are available in the GEO (http://www.ncbi.nlm.nih.gov/geo) and UALCAN (http://ualcan.path.uab.edu) repositories.

\section{Authors' contributions}

JZ and LX conceived the study. JW, ZL, KW, KZ and DX analyzed the data and drafted the manuscript. All authors reviewed and approved the final manuscript.

\section{Ethics approval and consent to participate}

Not applicable.

\section{Patient consent for publication}

Not applicable.

\section{Competing interests}

The authors declare that they have no competing interests.

\section{References}

1. Kamisawa T, Wood LD, Itoi T and Takaori K: Pancreatic cancer. Lancet 388: 73-85, 2016.

2. Michaud D: Epidemiology of pancreatic cancer. Minerva Chir 59: 99-111,2004.

3. Siegel R, Ma J, Zou Z and Jemal A: Cancer statistics, 2014. CA Cancer J Clin 64: 9-29, 2014.

4. Risch HA: Etiology of pancreatic cancer, with a hypothesis concerning the role of $\mathrm{N}$-nitroso compounds and excess gastric acidity. J Natl Cancer Inst 95: 948-960, 2003.

5. Kern SE, Shi C and Hruban RH: The complexity of pancreatic ductal cancers and multidimensional strategies for therapeutic targeting. J Pathol 223: 295-306, 2011.

6. Grasso C, Jansen G and Giovannetti E: Drug resistance in pancreatic cancer: Impact of altered energy metabolism. Crit Rev Oncol Hematol 114: 139-152, 2017.

7. Eskelinen M and Haglund U: Developments in serologic detection of human pancreatic adenocarcinoma. Scand J Gastroenterol 34 : 833-844, 1999.

8. Bass AJ, Lawrence MS, Brace LE, Ramos AH, Drier Y, Cibulskis K, Sougnez C, Voet D, Saksena G, Sivachenko A, et al: Genomic sequencing of colorectal adenocarcinomas identifies a recurrent VTI1A-TCF7L2 fusion. Nat Genet 43: 964-968, 2011.

9. Sjöblom T, Jones S, Wood LD, Parsons DW, Lin J, Barber TD, Mandelker D, Leary RJ, Ptak J, Silliman N, et al: The consensus coding sequences of human breast and colorectal cancers. Science 314: 268-274, 2006.

10. Wood LD, Parsons DW, Jones S, Lin J, Sjöblom T, Leary RJ, Shen D, Boca SM, Barber T, Ptak J, et al: The genomic landscapes of human breast and colorectal cancers. Science 318: 1108-1113, 2007.

11. Janky R, Binda MM, Allemeersch J, Van den Broeck A, Govaere O, Swinnen JV, Roskams T, Aerts S and Topal B: Prognostic relevance of molecular subtypes and master regulators in pancreatic ductal adenocarcinoma. BMC Cancer 16: 632, 2016.

12. Barrett T, Wilhite SE, Ledoux P, Evangelista C, Kim IF Tomashevsky M, Marshall KA, Phillippy KH, Sherman PM, Holko M, et al: NCBI GEO: Archive for functional genomics data sets-update. Nucleic Acids Res 41 (Database Issue): D991-D995, 2013.

13. Ritchie ME, Phipson B, Wu D, Hu Y, Law CW, Shi W and Smyth GK: Limma powers differential expression analyses for RNA-sequencing and microarray studies. Nucleic Acids Res 43: e47, 2015.

14. Zhang G, Schetter A, He P, Funamizu N, Gaedcke J, Ghadimi BM, Ried T, Hassan R, Yfantis HG, Lee DH, et al: DPEP1 inhibits tumor cell invasiveness, enhances chemosensitivity and predicts clinical outcome in pancreatic ductal adenocarcinoma. PLoS One 7: e31507, 2012.

15. Zhang G, He P, Tan H, Budhu A, Gaedcke J, Ghadimi BM, Ried T, Yfantis HG, Lee DH, Maitra A, et al: Integration of metabolomics and transcriptomics revealed a fatty acid network exerting growth inhibitory effects in human pancreatic cancer. Clin Cancer Res 19: 4983-4993, 2013. 
16. Xing Z, Chu C, Chen L and Kong X: The use of Gene Ontology terms and KEGG pathways for analysis and prediction of oncogenes. Biochim Biophys Acta 1860: 2725-2734, 2016.

17. Kanehisa M, Sato Y, Furumichi M, Morishima K and Tanabe M: New approach for understanding genome variations in KEGG. Nucleic Acids Res 47: D590-D595, 2019.

18. Kanehisa M, Furumichi M, Tanabe M, Sato Y and Morishima K: KEGG: New perspectives on genomes, pathways, diseases and drugs. Nucleic Acids Res 45: D353-D361, 2017.

19. Kanehisa M and Goto S: KEGG: Kyoto encyclopedia of genes and genomes. Nucleic Acids Res 28: 27-30, 2000.

20. Yu G, Wang LG, Han Y and He QY: clusterProfiler: An R package for comparing biological themes among gene clusters. OMICS 16: 284-287, 2012

21. Shannon P, Markiel A, Ozier O, Baliga NS, Wang JT, Ramage D, Amin N, Schwikowski B and Ideker T: Cytoscape: A software environment for integrated models of biomolecular interaction networks. Genome Res 13: 2498-2504, 2003.

22. Chin $\mathrm{CH}$, Chen SH, Wu HH, Ho CW, Ko MT and Lin CY: cytoHubba: Identifying hub objects and sub-networks from complex interactome. BMC Syst Biol 8 (Suppl 4): S11, 2014.

23. Chandrashekar DS, Bashel B, Balasubramanya SAH, Creighton CJ, Ponce-Rodriguez I, Chakravarthi BVSK and Varambally S: UALCAN: A portal for facilitating tumor subgroup gene expression and survival analyses. Neoplasia 19: 649-658, 2017.

24. Li B and Dewey CN: RSEM: Accurate transcript quantification from RNA-Seq data with or without a reference genome. BMC Bioinformatics 12: 323, 2011.

25. Wang X, Wang L, Mo Q, Dong Y, Wang G and Ji A: Changes of Th17/Treg cell and related cytokines in pancreatic cancer patients. Int J Clin Exp Pathol 8: 5702-5708, 2015.

26. Shen S, Gui T and Ma C: Identification of molecular biomarkers for pancreatic cancer with $\mathrm{mRMR}$ shortest path method. Oncotarget 8: 41432-41439, 2017.

27. Thomas PD: The gene ontology and the meaning of biological function. Methods Mol Biol 1446: 15-24, 2017.

28. Duffy MJ, Duggan C, Mulcahy HE, McDermott EW and O'Higgins NJ: Urokinase plasminogen activator: A prognostic marker in breast cancer including patients with axillary node-negative disease. Clin Chem 44: 1177-1183, 1998.

29. Nielsen TO, Andrews HN, Cheang M, Kucab JE, Hsu FD, Ragaz J, Gilks CB, Makretsov N, Bajdik CD, Brookes C, et al: Expression of the insulin-like growth factor I receptor and urokinase plasminogen activator in breast cancer is associated with poor survival: Potential for intervention with 17-allylamino geldanamycin. Cancer Res 64: 286-291, 2004.
30. Pavón MA, Arroyo-Solera I, Céspedes MV, Casanova I, León X and Mangues R: uPA/uPAR and SERPINE1 in head and neck cancer: Role in tumor resistance, metastasis, prognosis and therapy. Oncotarget 7: 57351-57366, 2016.

31. Amos S, Redpath GT, Dipierro CG, Carpenter JE and Hussaini IM: Epidermal growth factor receptor-mediated regulation of urokinase plasminogen activator expression and glioblastoma invasion via C-SRC/MAPK/AP-1 signaling pathways. J Neuropathol Exp Neurol 69: 582-592, 2010

32. Chaudhary A, Hilton MB, Seaman S, Haines DC, Stevenson S, Lemotte PK, Tschantz WR, Zhang XM, Saha S, Fleming T and St Croix B: TEM8/ANTXR1 blockade inhibits pathological angiogenesis and potentiates tumoricidal responses against multiple cancer types. Cancer Cell 21: 212-226, 2012.

33. Xu ZY, Chen JS and Shu YQ: Gene expression profile towards the prediction of patient survival of gastric cancer. Biomed Pharmacother 64: 133-139, 2010.

34. Liu P, Weng Y, Sui Z, Wu Y, Meng X, Wu M, Jin H, Tan X, Zhang $\mathrm{L}$ and Zhang Y: Quantitative secretomic analysis of pancreatic cancer cells in serum-containing conditioned medium. Sci Rep 6: 37606, 2016.

35. Borradori L and Sonnenberg A: Structure and function of hemidesmosomes: More than simple adhesion complexes. J Invest Dermatol 112: 411-418, 1999.

36. Järveläinen H, Sainio A, Koulu M, Wight TN and Penttinen R: Extracellular matrix molecules: Potential targets in pharmacotherapy. Pharmacol Rev 61: 198-223, 2009.

37. Yodsurang V, Tanikawa C, Miyamoto T, Lo PHY, Hirata M and Matsuda K: Identification of a novel p53 target, COL17A1, that inhibits breast cancer cell migration and invasion. Oncotarget 8: 55790-55803, 2017.

38. Chowdhury N and Sapru S: Association of protein translation and extracellular matrix gene sets with breast cancer metastasis: Findings uncovered on analysis of multiple publicly available datasets using individual patient data approach. PLoS One 10: e0129610, 2015.

39. Rizwan A,Bulte C,Kalaichelvan A, Cheng M,Krishnamachary B, Bhujwalla ZM, Jiang L and Glunde K: Metastatic breast cancer cells in lymph nodes increase nodal collagen density. Sci Rep 5: $10002,2015$.

40. Zou X, Feng B, Dong T, Yan G, Tan B, Shen H, Huang A, Zhang X, Zhang M, Yang P, et al: Up-regulation of type I collagen during tumorigenesis of colorectal cancer revealed by quantitative proteomic analysis. J Proteomics 94: 473-485, 2013. 